\title{
Flash pulmonary oedema: accurate evaluation of the renal arteries with multislice computed tomography
}

\author{
G Morgan-Hughes, A J Marshall, C Roobottom
}

Heart 2003;89:1251-1252

A subgroup of hypertensive patients, with preserved left ventricular function, are susceptible to recurrent episodes of sudden non-ischaemic pulmonary oedema caused by severe (usually bilateral) renal artery stenosis. Renal revascularisation may prevent the occurrence of "flash" pulmonary oedema. More than $70 \%$ of patients treated with percutaneous renal revascularisation are free from pulmonary oedema at 12 month follow up. ${ }^{12}$ Although patient selection for renal revascularisation remains controversial, it is generally accepted that patients with recurrent flash pulmonary oedema should at least be considered for intervention. ${ }^{3}$ Diagnosis requires non-invasive imaging of the renal arteries and this may be achieved with Doppler ultrasonography. However the failure rate is high and it is not therefore a reliable clinical technique. ${ }^{4}$ Magnetic resonance or computed tomography (CT) angiography are possible alternatives, and both provide additional imaging of the abdominal aorta and three dimensional visualisation. Magnetic resonance imaging is generally the less available of the two options. Helical, single slice, CT has been evaluated in comparison to invasive renal angiography, and the sensitivity and specificity for the detection of $\geqslant 70 \%$ stenoses, at all sites, has been estimated at up to $92 \%$ and $83 \%$, respectively (using maximum intensity projection). ${ }^{5}$ Multislice CT, which is now widely available, has improved spatial and temporal resolution. We have studied thin section, multislice CT and comparative invasive renal angiographic (as gold standard) data with a view to establishing the accuracy of this technique.

\section{METHODS}

In this retrospective observational study we identified all patients who had undergone assessment for possible renal artery stenosis in the preceding 12 months. A total of 18 patients had comparative invasive and thin section, multislice CT renal angiographic data available for comparison, and these patients formed the study group. Thin section multislice CT (Philips MX8000, Philips Medical Systems, UK) was acquired using $140 \mathrm{kV}, 200 \mathrm{mAs} / \mathrm{slice}$, a collimation of $4 \times 1.0 \mathrm{~mm}$, increment 0.6 , and a pitch of 1.25 with appropriate $\mathrm{z}$ axis coverage. Contrast enhancement was achieved with $100 \mathrm{ml}$ of iodinated contrast (iopromide) via an 18 gauge antecubital vein catheter at $3 \mathrm{ml} / \mathrm{s}$ triggered using bolus tracking. Images were transferred to a workstation (MxView, Philips Medical Systems, UK) for evaluation, which was completed using axial data, maximum intensity projections, and three dimensional reconstructions manipulated with volume rendering techniques (fig 1). All multislice CT data were evaluated, by one investigator, before invasive evaluation and therefore blind to the findings of the gold standard test. The invasive angiograms (Philips Integris Allura, Philips Medical Systems, UK) were carried out by experienced operators, using standard techniques. Findings from invasive angiography were then compared with the original thin section, multislice CT for the presence or absence of $\geqslant 50 \%$ and $\geqslant 70 \%$ stenoses, at any site.

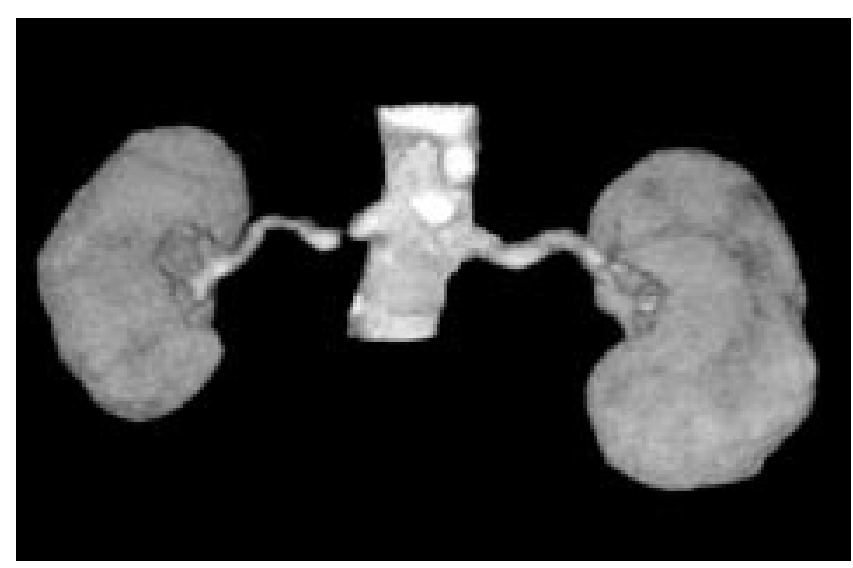

Figure 1 Three dimensional reconstruction of a thin section, multislice $\mathrm{CT}$ renal angiogram. Flash pulmonary oedema is usually caused by severe, bilateral disease. This angiogram shows a unilateral severe $(>90 \%)$ proximal renal artery stenosis on the right. The right kidney is reduced in size.

\section{RESULTS}

Multislice CT acquisition time varied between 12-15 seconds, evaluation was rapid, and there were no complications. A total of 58 main and accessory renal arteries were demonstrated with invasive angiography. Of these vessels 98\% (57/58) were correctly identified and assessable with thin section, multislice CT. The one vessel that could not be fully assessed arose from the internal iliac artery, which is outside the usual acquired scan volume. At catheter angiography, at all sites, 18 lesions of $\geqslant 50 \%$, and 16 lesions of $\geqslant 70 \%$ stenosis were diagnosed. All 57 vessels were correctly judged, with thin section, multislice CT, for the presence or absence of a lesion causing $\geqslant 50 \%$ stenosis $($ sensitivity $=$ specificity $=100 \%$ ). One $70 \%$ lesion was under scored, and graded at $60 \%$. Therefore for the accurate detection of $\geqslant 70 \%$ stenosis we found a sensitivity of $94 \%$, a specificity of $100 \%$, negative predictive value $98 \%$, and positive predictive value $100 \%$. All total occlusions (four) were correctly identified.

\section{DISCUSSION}

Previous, single slice, CT evaluation of renal arteries has been carried out with major scan parameters ( $3 \mathrm{~mm}$ collimation) giving a lower spatial resolution than is now available. ${ }^{5}$ Even the improvements offered by four slice acquisition have been superseded in many centres by the installation of 16 slice scanners. However, we have demonstrated that thin section, four slice CT renal angiography is sufficiently accurate that invasive diagnostic renal angiography is no longer necessary. Invasive procedures can be reserved for planned intervention. Prior three dimensional CT visualisation of both the renal arteries and the aorta may facilitate the procedure. Furthermore recent data assessing renal artery stent lumen diameters at multislice CT angiography, with catheter angiography, 
suggests that thin section multislice CT angiography may also be used for stent follow up. ${ }^{6}$

The potentially negative aspects of CT renal angiography are the patient radiation dose, which is comparable to catheter angiography, and the nephrotoxicity of iodinated contrast. Concerns over radiation are less relevant in what is generally an older population, and although nephrotoxicity is a genuine risk, it rarely leads to a clinically relevant deterioration in renal function.

Renal angiography, whether invasive or not, should be performed in order to identify renal artery stenosis that would be treated if found. Revascularisation of atherosclerotic, stenosed, renal arteries is not always beneficial. The results of ongoing studies may help identify the subgroups of patients with atherosclerotic renal artery stenosis who benefit the most from revascularisation. Until this time most authors recommend revascularisation for rapidly progressive renal failure with no other obvious cause, severe refractory hypertension, and recurrent flash pulmonary oedema. ${ }^{78}$ As subspecialisation increases the patient group most likely to be referred to a cardiologist for investigation is the group with recurrent flash pulmonary oedema. It is therefore important that cardiologists investigating these patients should be aware of the accuracy and availability of this rapid and safe method of non-invasive renal artery assessment.

\section{ACKNOWLEDGEMENTS}

The authors are undertaking research into the clinical applications of cardiac computed tomography. The work is partially supported by a grant from Philips Medical systems.

\section{Authors' affiliations}

G Morgan-Hughes, A J Marshall, Cardiology Department, South West Cardiothoracic Centre, Plymouth NHS Trust, Derriford, Plymouth, UK

C Roobottom, Department of Radiology, Plymouth NHS Trust

Correspondence to: Dr G J Morgan-Hughes, Cardiology Department, South West Cardiothoracic Centre, Plymouth NHS Trust, Derriford, Plymouth PL6 8DH, UK; hughesgi@talk2 1.com

Accepted 13 March 2003

\section{REFERENCES}

1 Khosla S, White C, Collins T, et al. Effects of renal artery stent implantation in patients with renovascular hypertension presenting with unstable angina or congestive cardiac failure. Am J Cardiol 1997;80:363-6.

2 Bloch M, Trost D, Pickering T, et al. Prevention of recurrent pulmonary odema in patients with bilateral renovascular disease through renal artery stent placement. Am J Hypertens 1999;12:1-7.

$3 \mathrm{Lim}$ S, Rosenfield K. Renal artery stent placement: indications and results. Curr Interv Cardiol Rep 2000;2:130-9.

4 Safian R, Textor S. Renal-artery stenosis. N Engl J Med $2001 ; 344: 431-42$.

5 Rubin G, Dake M, Napel S, et al. Spiral CT of renal artery stenosis: comparison of three-dimensional rendering techniques. Radiology 1994; 190:181-9

6 Behar J, Nelson R, Zidar J, et al. Thin-section multidetector CT angiography of renal artery stents. Am J Roentgenol 2002;178:1155-9.

7 Martin L, Rundback J, Sacks D, et al. Quality improvement guidelines for angiography, angioplasty, and stent placement in the diagnosis and treatment of renal artery stenosis in adults. J Vasc Interv Radiol 2002;13:1069-83

8 Geddes C, Jardine A. Diagnosis and treatment of atherosclerotic renal artery stenosis (ARAS). Minerva Urol Nefrol 2002;54:29-36.

\section{IMAGES IN CARDIOLOGY}

\section{Giant right atrial thrombosis associated with Hickman catheter}

$\mathrm{H}$ ickman catheters were introduced in the 1970s as long term central venous catheters for prolonged parenteral nutrition; their applications have been extended for the purpose of chemotherapy, bone marrow transplantation, and long term intravenous access for other reasons. The results of the use of Hickman catheters have been satisfactory, but complications are also common.

A right atrial thrombus was detected during routine echocardiograhic evaluation for anthracycline treatment in a 15 year old female patient with acute lymphoblastic leukaemia. She was treated with tissue plasminogen activator ( $\mathrm{t}-\mathrm{PA}$ ) initially but underwent surgery because of an unsatisfactory response. She had a giant atrial thrombosis in her right atrium. The thrombus was successfully excised and the badly damaged endocardium was plicated to avoid recurrence.

This case illustrates the importance of placing the tip of catheter at the junction of the lower superior vena cava and right atrium in order to avoid endothelial damage.

S Kula

A Saygili

S F Tunaoglu

$R$ Olgunturk kula_s@yahoo.com

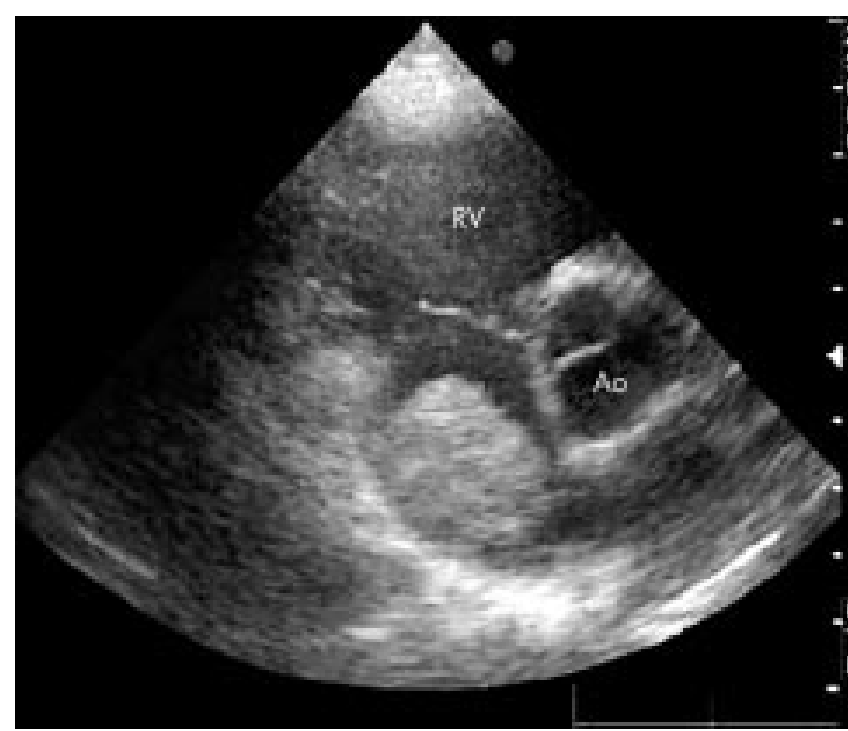

Giant right atrial thrombus in parasternal short axis view. 